\title{
STUDIES ON MASK-LESS ELECTROCHEMICAL ADDITIVE MANUFACTURING
}

This paper was downloaded from TechRxiv (https://www.techrxiv.org).

\section{LICENSE}

CC BY 4.0

SUBMISSION DATE / POSTED DATE

$25-06-2021 / 30-06-2021$

CITATION

Bhardwaj, Abhishek (2021): STUDIES ON MASK-LESS ELECTROCHEMICAL ADDITIVE MANUFACTURING. TechRxiv. Preprint. https://doi.org/10.36227/techrxiv.14850306.v1

$\mathrm{DOI}$

10.36227/techrxiv.14850306.v1 


\title{
STUDIES ON MASK-LESS ELECTROCHEMICAL ADDITIVE MANUFACTURING
}

\author{
ABHISHEK BHARDWAJ \\ Department of Mechanical Engineering \\ Kalinga Institute of Industrial Technology, Bhubaneswar \\ Odisha -751024
}

\section{ABSTRACT}

Additive Manufacturing (AM) of metallic structures is a thermal process of layer by layer metal addition manufacturing process, produces parts directly from $3 D$ CAD models. In this manufacturing process localized electrochemical deposition combines with the additive manufacturing method to manufacture metal parts at room temperature. In this paper, the feasibility of Mask-less Electrochemical Additive Manufacturing (ECAM), as a non-thermal process is studied. Layer by layer deposition has been done using electrochemical tip to manufacture nickel microstructures. Simultaneously pulse wave characteristics and their effects on deposition has been studied. Localized electrodeposition (LED) was explored as an AM process with high control over process parameters and output parameters. The localization of electrodeposition is carried out by using Ultra micro electrodes (UME) and low throwing power electrolytes. Variation in some process parameters such as voltage and electrode gap are found to have high influence in output parameters such as thickness. The simulations can predict the output width of deposition of experiments with an error of 8$30 \%$, so it has potential to apply as an additive manufacturing technique of complex three-dimensional (3D) parts on the microscale.

Keywords: localize electrodeposition

\section{NOMENCLATURE}

$\begin{array}{ll}h_{0} & \text { maximum theoretical deposition thickness } \\ i_{\text {avg }} & \text { average applied current density } \\ t & \text { elapsed deposition time } \\ Z & \text { charge number } \\ M & \text { molar mass } \\ F & \text { Faraday constant } \\ \rho & \text { density } \\ i_{0} & \text { current density }\end{array}$

$\begin{array}{ll}V_{M} & \text { molar volume of deposit } \\ R & \text { ideal gas constant } \\ T & \text { temperature in } \mathrm{K} \\ \alpha & \text { cathodic charge transfer coefficient } \\ \eta & \text { overpotential } \\ C & \text { instantaneous concentration of nickel cation } \\ x & \text { distance from the cathode } \\ D & \text { diffusivity of the cation } \\ C_{\infty} & \text { bulk electrolyte concentration } \\ n & \text { number of electrons transferred per metal }\end{array}$

\section{INTRODUCTION}

AM is an approach that is used to manufacture layer by layer metallic complex 3D shapes directly from computer generated CAD models. These parts have numerous applications in various fields such as medical, biotechnology, jewelry, optics, electronics, automotive and aerospace industries. To fulfill these demands several new micro manufacturing technologies are risen up such as electroplating, lithography, laser ablation, plating, photolithography, molding, chemical etching, and additive manufacturing. However process time, choice of material, porosity, internal stress, scalability, part errors, fatigue performance and strength are some general limitations of AM. The wide use of these processes are limited in industries due to lack of confidence in material properties of these parts. While selective laser beam and electron beam melting products suffer high residual stress due to complete melting of metals, capable to produce high quality parts than machined parts. Earlier 3D features in micro manufacturing and nano manufacturing are distant-dream, limited to 2D and 2.5D features [Ambrosi Adriano et al. 2016]. AM as a recently developed process has the capability of producing complex 3D parts manufacturing from 
computer generated CAD models with various materials such as metals, polymer and piezoceramic materials.

ECAM has potential to overcome some of these limitations as it is a non-thermal process that has considerably lower residual stresses and the addition of material is atom by atom resulting in excellent microstructural properties which can be controlled in process. One major advantage is the mask-less nature achieving shorter development times, compared to conventional postprocessing and rapid prototyping procedures [M. Murali et al. 2015]. ECAM is capable of depositing most of the conductive materials such as metals, metal alloys, semiconductors and conductive polymers. But LED is challenging in ECAM. Electrodeposition techniques offer significant technical and economic advantages characteristic to the electroplating technology. Electroplating and electroforming processes are capable of depositing materials over large area but localization is not possible. Using micro tip electrode anode, ECAM is capable of creating microstructures such as metal pillars through LED[Brant A. et al. 2015].

There are some unique advantages in ECAM. To advance the nano ECAM process to make it predictable, reliable and commercially-viable additive manufacturing process requires deep understanding of electrical and chemical parameters that are linked in this metal addition process [Volgina $\mathrm{V}$ M et al. 2018]. So, in this paper the feasibility of mask-less ECAM of metal parts made of nickel using layer-by-layer localized electrochemical deposition is studied. Input-output relationships are established, and verified with experimental results. The effects of process parameters such as voltage, electrolyte concentration, and interelectrode gap have been studied to establish appropriate settings for the process.

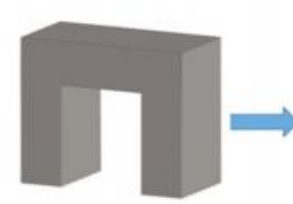

3D CAD Model

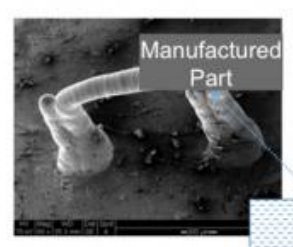

Electrochemical cell

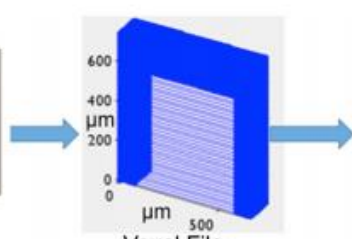

Voxel File

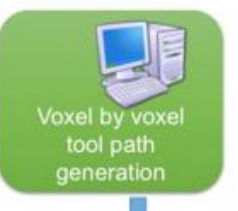

generation

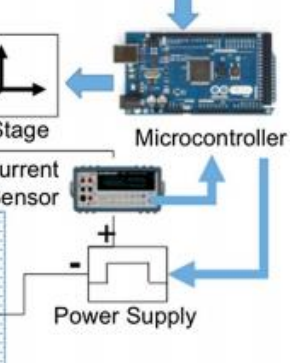

Figure 1 - Overview of the electrochemical additive manufacturing process [ Kamaraj Abishek B. et al. 2017]

\section{METHODS OF NICKEL BASED ECAM}

Mask-less ECAM of metal parts made of nickel microstructures using layer by layer electrodeposition is studied. The major solution is focused on localization of the metal

deposits. LED can be done by using various technologies such as micro tip electrode of nickel.

AM techniques have limitations such as choice of material, anisotropy, porosity, process time, strength, scalability, support structure, and internal stresses. Though AM can produce various complex parts, but residual has impacts on wall thickness. There are other limitations too such as powder handling, part removal, surface roughness, and resolution. For selective laser melting process, there is no specialized process for micro manufacturing or nano manufacturing [Sundaram Murali M et al 2015]. The other limitations related to AM process are binder injection, jet processing, layer binding, resolution, and surface finish of the produced 3D microstructures. Binders get clogged on injection path and jet processing should be done in a low oxygen environment to prevent the formation of a surface oxide layer thus resulting in changes to the physical properties of the jet surface.

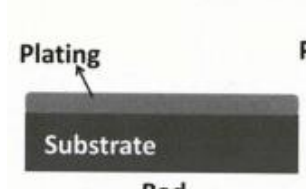

Bad

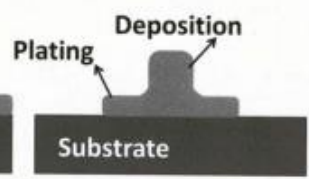

Obtained

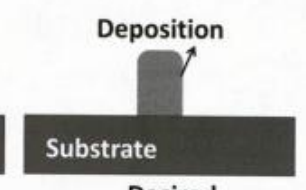

Desired
Figure 2 - Cross-sectional view of the different outcomes of ECAM process [M. Murali et al. 2015]

There are various process parameter that affect the characteristic of the deposited structures and the deposition rate. The potential applied between the electrodes needs to be above a threshold for deposition to occur. But very high voltages can create porous or irregular deposition structures, due to the depletion of ions at high currents, and bubble formation. Studies says that there is no effect of choice of electrolytes on electro deposition process [Sundaram Murali M et al. 2015]. But the quality of deposition gets affected by low concentration of electrolytes. The choice of electrolyte for the electrodeposition mostly depends on the electrolyte used for electroplating the same material. The localized deposition can be improved by insulation of micro tip [Brant A. et al. 2015]. To get a stable electrode gap is challenging. Some of the factors that affect the quality of deposition are voltage, pulse period, electrolyte concentration, tool electrode speed, electrode shape, electrolyte circulation, and additives in the electrolyte. Duty cycle also effects on the porosity and surface finish of the parts [V.M. Volgin et al. 2018]. As duty cycle decreases porosity also decreases, resulting smoother surface finish. The optimum duty cycle range that shows best result is 0.4-0.5.

Throwing power of an electrolyte can be defined as the ability of an electrolyte to evenly coat during electrochemical deposition process [V.M. Volgin et al. 2018]. A high concentration and low electrolytic resistance electrolyte usually have a high throwing power. $p H$ is also a factor of throwing power in localized electrodeposition [Sundaram Murali M et al. 2015]. Higher $p H$ value electrolytes are expected to have higher throwing power. But for mask less ECAM lower $p H$ value 
electrolytes are preferred for lower throwing power and better localize electrodeposition.

Various factors that affect the deposition of metals on a substrate include mass transfer, electron transfer, electrical and chemical potential and crystal growth [V.M. Volgin et al. 2018]. Three mechanisms that come up with metal deposition are diffusion, migration and convection. Diffusion, migration and convection are driven out by electrical gradient, electrical potential gradient and pressure gradient. In many studies, numerical modelling of electro deposition is mainly focused on rate of deposition but not on migration of electrons.

\subsection{Design parameters and formulae}

According to Faraday's law, the maximum theoretical deposition thickness can be described as a function of average current density and total elapsed deposition time [Kamaraj Abhishek et al. 2016].

$$
h_{0}=\frac{M_{N i} i_{a v g} t}{\rho_{N i} Z_{N i 2+} F}
$$

Here, the current efficiency is assumed to be $100 \%$. $\mathrm{h}_{0}$ is the maximum theoretical deposition thickness, $i_{\text {avg }}$ is the average applied current density, $\mathrm{t}$ is the elapsed deposition time, $\mathrm{ZNi}^{2+}$ is the charge number for $\mathrm{Ni}^{2+}$ ions (equal to 2), $\mathrm{M}_{\mathrm{Ni}}$ equals the molar mass of nickel $(58.71 \mathrm{~g} / \mathrm{mol}), \mathrm{F}$ is the Faraday constant $(9.649 \mathrm{C} / \mathrm{mol})$, and $\rho_{\mathrm{Ni}}$ is the density of nickel $\left(8.9 \mathrm{~g} / \mathrm{cm}^{3}\right)$.

A decrease in grain size can be achieved by increasing pulse width by varying the pulse period and holding duty cycle and ontime constant. The following relation describes deposit cluster size as a function of current density.

$$
d(t)=2\left(V_{M} /_{Z F}\right)\left(i_{o} f(\eta)\right) t
$$

This relation can give clues to resulting grain size where $\mathrm{i}_{0}$ is the current density in $\mathrm{A} / \mathrm{cm}^{2}, \mathrm{~V}_{\mathrm{M}}$ is the molar volume of the deposit in $\mathrm{cm}^{3} / \mathrm{mol}, \mathrm{z}$ is the charge number ( 2 for $\mathrm{Ni}^{2+}$ ions) and $f(\eta)$ is the over potential function, which is described by

$$
f(\eta)=\exp \left(\frac{\alpha x F n}{R T}\right)-\exp \left(\frac{(1-\alpha) z F n}{R T}\right)
$$

Fick's law of diffusion for one dimensional mass transfer governing equation for nickel diffusion is given by

$$
\frac{\partial C}{\partial t}=D \frac{\partial^{2} C}{\partial x^{2}}
$$

Therefore, these parameters have to be controlled for LED which gives a better result for ECAM.

\section{SIMULATION SET UP}

Simulation of LED process is done by modelling the electrolyte itself as a two dimensional cross section at the center of the tool. A perfectly square mesh is used in the region between the tool and the substrate to ensure consistency in calculations. The electrolyte is modeled as conductive medium with resistivity value $\rho$ at a given concentration. This $\rho$ value is different from the previously mentioned $\rho_{N i}$ value.

The geometrical parameters of the simulation are tool diameter (D), tool width and internal gap between tool and substrate $(\mathrm{g})$. The region of electrolyte is taken as 5D on each side of the electrode, beyond which current density effects are negligible. The radius $r_{f}$ of the tool is set to equal one tenth of the tool width. Then, a voltage value is applied to the bottom and curved sides of the tool, and another voltage value is applied to the substrate, denoted as $V_{\text {tool }}$ and $V_{\text {substrate }}$ respectively as shown in table 1 .

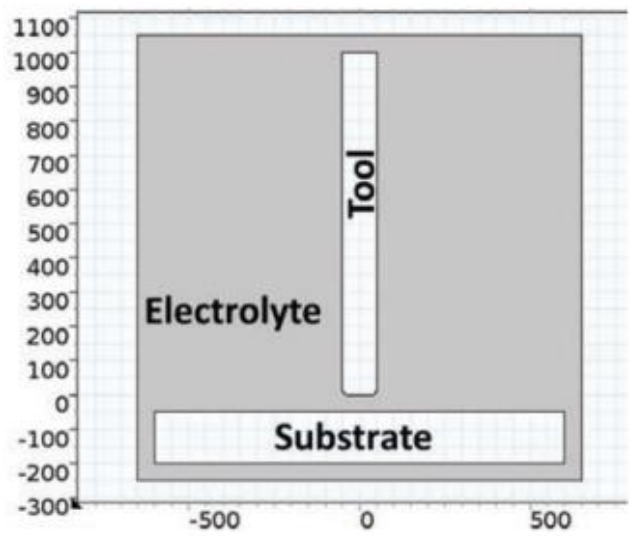

Figure 3 - Geometry of the simulation space (axis unit $\mu \mathrm{m}$ ) [ Kamaraj Abishek et al. 2016]

These conditions are solved by considering it as first iteration. The voltage is simulated to be applied over an iteration time $\mathrm{t}_{\text {iter }}$ and a duty cycle $\mathrm{d}$. The bottom surface is divided into $\mathrm{n}$ elements from bottom to top and isolated them. Equation 1 is needed to modify for all the elements i from 0 to $n$.

$$
h_{i}=\frac{M_{N i} i_{\text {avg }} t_{i t e r}}{\rho_{N i} Z_{N i 2+} F}
$$

All elements in the same column as the element are then investigated. If any element's y position is less than the calculated hj for the bottom element of the column, then the element is "killed." This means that all material properties of the element are switched to zero and the element is effectively nonexistent. A killed element represents the nickel deposit replacing the electrolyte at that location. The summary of material modelling parameters are listed in table 1 .

Applying boundary and initial conditions to equation no. 4

$$
\begin{aligned}
& C(x, 0)=C_{\infty} \\
& C\left(x_{G}, t\right)=C_{\infty}
\end{aligned}
$$

$$
\left.\frac{\partial C}{\partial x}\right|_{x=0}=\frac{i_{0}}{n F D}\left[\frac{C_{(0, t)}}{C_{\infty}} 10^{\left(\frac{\alpha_{C} F}{2.3 R T} \eta\right)}-10^{-\left(\frac{\alpha_{C} F}{2.3 R T} \eta\right)}\right]
$$


The effect of ion migration and electrolyte fluid motion effects are considered as negligible in the model as electrolyte flow is not imposed in micro-ECAM [Brat A. et al. 2015]. This partial derivative equation is discretized into ordinary differential equation with 200 nodes and solved using a commercially available differential equation solver. The solution gives special distribution of ionic species over the inter-electrode gap and over time. The current density can be solved using flux density at $x=0$ using the equation no. 9

$$
i(t)=n F D \frac{\partial C}{\partial x}
$$

\begin{tabular}{lc}
\hline \hline Input parameter & Levels \\
\hline$D(\mu \mathrm{m})$ & $10,50,100,250$ \\
$g(\mu \mathrm{m})$ & $D, 2 D, 3 D$ \\
$V_{\text {tool }}(\mathrm{V})$ & $3,4,5$ \\
$d(\%)$ & $25,50,75$ \\
$\rho(\%$ of default value $)$ & $50,100,200$ \\
\hline \hline
\end{tabular}

Table 1: Summary of material modelling parameters [Brant A et al. 2015]

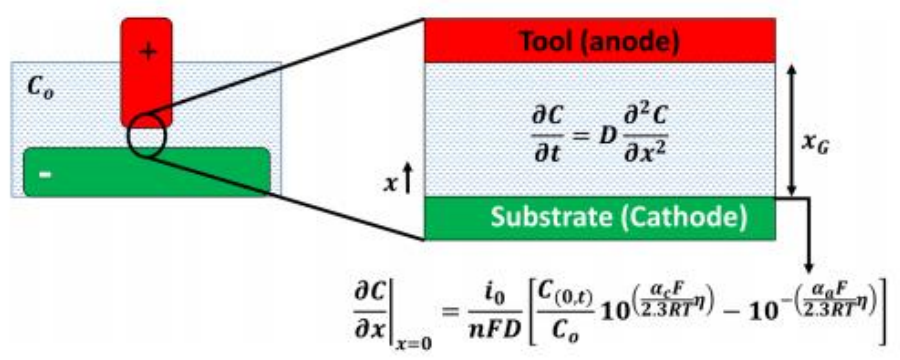

Figure 4 - Schematic showing the model setup and boundary conditions [ Kamaraj Abishek B. et al. 2017]

\section{SIMULATION RESULTS}

The results are varying with varying input parameters in each level. These results are represented as different varying graphs over corresponding conditions and iterations.

\subsection{Effect of varying tool width on resulting deposition:}

Varying tool width had more significant effects on the resulting deposition than any other parameters. When the tool width was increased, this was seen to increase the overall deposition width and quality and decrease the overall rate and average peak current density [Brant A et al. 2015]. This is because if the gap is maintained at a value of D, increasing the tool width also increases the gap. Therefore, current effects are weaker for larger tool sizes and larger gap sizes, yielding less of a maximum current and a lower deposition rate.

The reduction in tool size results in overall increase in deposition. This is also correlated with current density. When tool size decreases current density value increases. But after deposition, results showed that smallest tool results very poor quality deposition electrodeposition [Sundaram Murali M et al. 2015]. There is an optimal tool size of $1.5 \AA$ radius for best quality of deposition. Increase in tool radius results in a decrease in quality. It is due to a wider distribution of the electric field. Decrease in radius also results in a decrease in quality. It is due to less atoms present to exert an electrostatic force on the cations, even though the smallest tool would have had the strongest induced charge and electric field per atom [Shetty D et al. 2012].

\subsection{Effect of varying gap on resulting deposition:}

The shortest inter electrode gap results in fastest and rapid deposition of metals but the quality of deposition is very poor and short lived. The highest quality of electrodeposition is achieved with middle interelectrode gap. A further increase in height results in a slower deposition, lower current density and lower quality [Brant A et al. 2015]

When the tool width was increased, this increase the overall deposition width and quality and decrease the overall rate and average peak current density. This is because if the gap is maintained at a value of $\mathrm{D}$, increasing the tool width also increases the gap. Therefore, current effects are weaker for larger tool sizes and larger gap sizes, yielding less of a maximum current and a lower deposition rate [Sundaram Murali $\mathrm{M}$ et al. 2015]. At larger inter-electrode gaps, the effect of the pulse voltage becomes negligible as the current density does not vary a lot between the pulse-on and off times [Kamaraj Abhishek. B. et al. 2017]

\subsection{Effect of varying tool voltage on resulting deposition:}

Variation of the tool voltage resulted in a less drastic change of output deposition qualities than that of varying the tool width and gap. A greater applied voltage resulted in an increase in deposition rate, quality, width, and peak current density [Sundaram Murali M et al. 2015].

Tool voltage has direct relationship to tool initial deposition speed and initial current density. But final current density follows an inverse relationship. This is due to the more ion depletion from a higher potential difference [Brant A et al. 2015].

\subsection{Effect of varying electrolyte concentration on resulting deposition:}

Variation of the electrolyte concentration, expressed as a change in resistivity, was seen to affect the current density. A lower resistivity led to a higher initial peak current density [Sundaram Murali M et al. 2015]. 
Current density and deposition speed values are lowest at the extreme points i.e. at highest and lowest points. However these output parameters were highest for the middle concentration values [Brant A et al. 2015]. Due to the increased in resistance, the highest concentration of electrolyte gave the highest quality deposit despite having the slowest deposition speed and lowest output current density.

\subsection{Effect of varying duty cycle on resulting deposition:}

Increase in duty cycle was seen to have effects in increasing the deposition quality and width [Sundaram Murali M et al. 2015]. As expected, changing the duty cycle had no effect on the resultant current density since the current density is dependent on resistivity, geometry of the medium, and voltage [Brant A et al. 2015].

Lower duty cycle $(25 \%)$ had the highest steady state current density because of the longer the pulse-off time. However, at lower duty cycles, the average current density value is still lower due to long pulse-off time. This might result in slower deposition rates. Duty cycle beyond $75 \%$ seems to perform similarly to DC current conditions [Kamaraj Abhishek B. et al. 2017]. Thus, a balance between pulse-on and pulse-off time is to be optimized to maximize steady-state current while still replenishing the ions in the inter-electrode region.

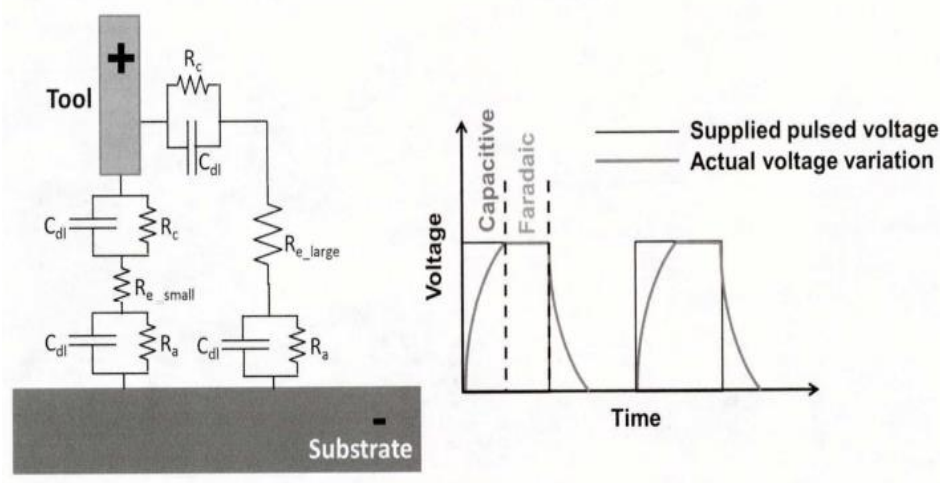

Figure 5 - Electric equivalent circuit for deposition [M. Murali et al. 2015]

\section{EXPERIMENTAL SET-UP}

The experimental set up for ECAM consists of three axis CNC stage and controller and a electrolyte tank, fitted on top of the stage, acting as the build chamber. Three individual NEMA 08 stepper motors that controls the 3 axes motion of the process, which are in turn connected to a parallel port breakout board. This breakout board connects to the PC and acts as a liaison between the stage and the PC based controller. Mach3 is the PC based controller, which is used to control the movement of the axes of the stage. It takes in G-codes and sends out step direction pulses based on the G-codes to control the movement of the stage.
The maximum travel distance on each axis is $10 \mathrm{~mm}$. The minimum travel distance on each axis is $1 \mu \mathrm{m}$. Feed rate is $32 \mathrm{~mm} / \mathrm{min}$ to $1 \mu \mathrm{m} / \mathrm{s}$. The spindle is mounted over the electrolyte tank which houses the tool required for the machining process. This spindle is controlled by Mach3 controller. The feedback for any contact is taken as an input into the instrument control software to ensure a constant gap between tool and the substrate at each deposition layer. In-house software and hardware were developed and used in order to implement layered manufacturing of the part starting with only the STL file as the input from a CAD system.

The electrolyte used in the process contains nickel sulfate (240 g), nickel chloride (45 g), and Boric acid (30 g) per liter of distilled water as this has a lower throwing power than other nickel deposition electrolytes.

An equivalent electrochemical circuit shows the representation of double layer capacitance $\left(C_{d}\right)$ and electrolyte resistance $\left(R_{c}\right)$. The double layer makes the electrochemical cell effectively an RC circuit with a time constant $\tau$ related to the resistance which is a function of electrolyte concentration and interelectrode gap and to the double layer capacitance. The rise time for an $\mathrm{RC}$ circuit is given as $2.2 \tau$ and during this time period the current is capacitive in nature and no deposition occurs. Duty cycle has serious effect on deposition. Higher and lower duty cycle showed inefficient deposition because of the pulse on time which was not longer than the time constant resulting in capacitive currents only.

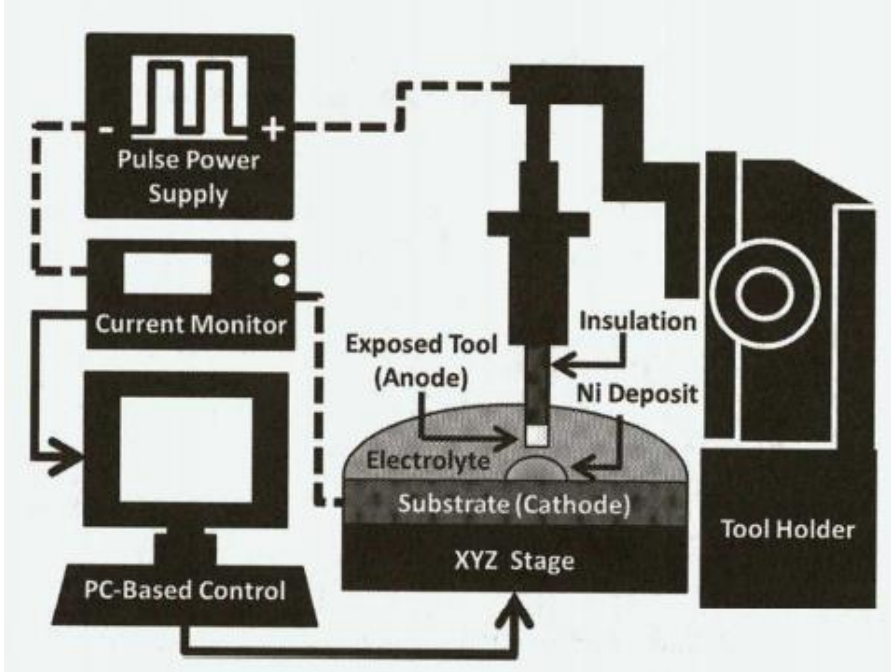

Figure 6-ECAM experimental set up [Brant A. et al. 2015]

\section{RESULTS AND DISCUSSIONS}

To establish the appropriate process parameters, such as the voltage, pulse settings, and interelectrode gap etc. the preliminary experiments were conducted. The quality of nickel deposits were analyzed using a surface profilometer and an optical microscope. Using electrolytes having $p H$ value greater than 5 and large interelectrode gaps which is greater than 100 $\mu \mathrm{m}$, resulted in predominant plating and no localized deposits were formed. Localization was achieved only when the gap was 
kept as low as possible, along with a low throwing power electrolyte. Even then, some plating was observed but was negligible in comparison to the deposit material size.

To establish the capability of ECAM system, 3D free hanging metal structures were built using this system. A shape resembling the number " 7 " with height $600 \mu \mathrm{m}$ and hanging portion length $600 \mu \mathrm{m}$ was fabricated using the system. The average diameter of the structure was in the range of 80-100 $\mu \mathrm{m}$. Using CNC controlled layered localized mask-less electrochemical deposition, layer-by-layer manufacturing of nickel microstructure was created in an in-house built CNC stage. CAD based layer-by-layer manufacturing allows for creation of more complex parts. Initially a simple straight line has drawn to test the process. In figure 7 shows the optical microscope image of a straight line that was manufactured usig the mentioned parameters. The length of the line is $1 \mathrm{~mm}$ and height of each layer is $10 \mu \mathrm{m}$. Initial gap between electrodes kept as $10 \mu \mathrm{m}$. Figure 8 show the optical microscope image of a " $U$ " shaped geometry that was layer manufactured using the same set of process parameters. The part program for "UC" shaped geometry with ten layers was used cab be seen in the figure 9 with showing dimensions. The distance between the two geometries was kept at $1 \mathrm{~mm}$.

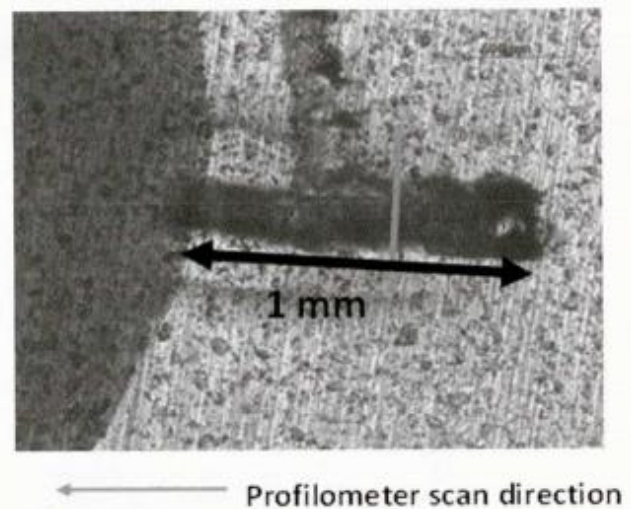

Figure 7 - Optical microscope image of straight line geometry and profilometer scan [M. Murali et al. 2015]

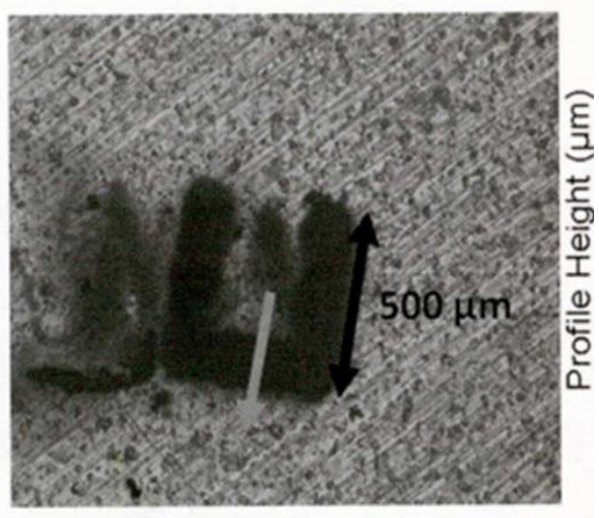

\section{Profilometer scan direction}

Figure 8 - Optical microscope image of U shaped geometry and profilometer scan [M. Murali et al. 2015]
With the appropriate process parameters established for the continuous deposition, the AM process has to implement as discussed above to directly manufacture a part from its STL file. The part model and the optical microscope image of the deposit are given in the figure 10. The final results shows that the size of the deposit is slightly smaller than the tool size especially with curved features. A part with 30 layers was manufactured with each layer thickness $10 \mu \mathrm{m}$ using tool size of $100 \mu \mathrm{m}$, as shown in figure 11 .

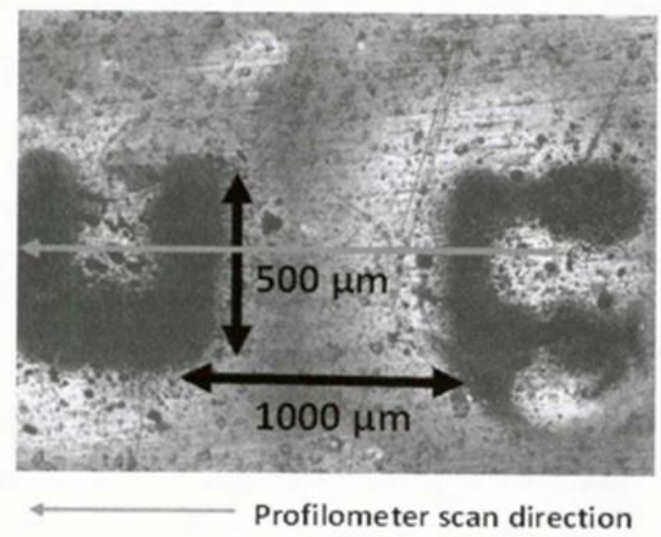

Figure 9 - Optical microscope image of UC shaped geometry and profilometer scan [M. Murali et al. 2015]
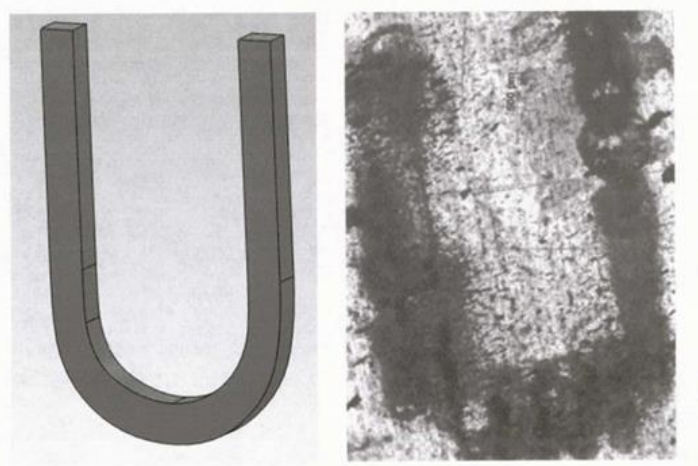

Figure 10 - (Left) part model and (right) the two layer deposit of the part [M. Murali et al. 2015]

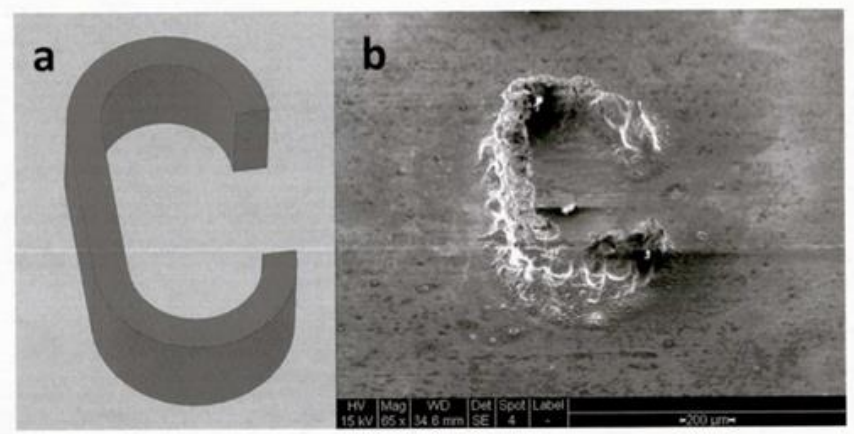

Figure 11 - (a) CAD model of letter " $C$ " to be manufactured in 30 layers and (b) SEM image of electro chemically additive manufactured part [M. Murali et al. 2015] 


\section{CONCLUSIONS}

The detailed study on feasibility of ECAM, various factors and parameters of ECAM process have been done. The findings are listed below as conclusions -

- ECAM system capable of manufacturing nickel microstructures from a CAD model has been built.

- As a non-thermal process it has the major advantage of having considerably lower residual stresses.

- Addition of material is a chemical process, atom by atom resulting in excellent microstructural properties.

- The process parameters that influence the deposition were analyzed with the interelectrode gap, tool size, voltage, electrolyte concentration and the pulse setting influencing the size and quality of the deposition.

- These results also helps to determine the relationships between the parameters of electrodeposition and the shape and dimensions of formed microstructures.

- This method has potential applications in the biomedical industry for designed micro-implants, in the jewelry industry for intricate shape manufacture, microgears, micromotors, and electronics industry.

- But this method is not effective in lowering the process time. AM processes are still facing challenges to reduce the process time for effective industry use.

\section{ACKNOWLEDGEMENTS}

I would like to take this opportunity to thank our course instructor and seniors for motivating us to explore

various research areas and providing us the opportunity to present this term paper.

\section{REFERENCES}

[1] Ambrosi Adriano, Pumera Martin, 2016 "3D-printing technologies for electrochemical applications", Chem. Soc. Rev.

[2] Brant Anne, Sundaram Murali, 2019 "Molecular dynamics study of direct localized overpotential deposition for nanoscale electrochemical additive manufacturing process", Precision Engineering 56, 412-421

[3] Brant A., Sundaram M., and Kamaraj A. B., 2015 "Finite Element Simulation of Localized Electrochemical Deposition for Mask-Less Electrochemical Additive Manufacturing," ASME J. Manuf. Sci. Eng.

[4] Kamaraj Abishek, Lewis Spenser, Sundaram Murali, 2016 "Numerical Study of Localized Electrochemical Deposition for Micro Electrochemical Additive Manufacturing", ISEM XVIII
[5] Kamaraj Abishek B, Sundaram Murali, 2017, “A study on the effect of inter-electrode gap and pulse voltage on current density in electrochemical additive manufacturing", Journal of Applied Electrochemistry

[6] Shetty D., and Ly D., 2012 “Additive Manufacturing: Exploration of Porosity and Form Features Using Layer by Layer Deposition,” ASME J. Manuf. Sci. Eng.

[7] Sundaram Murali M., Kamaraj Abishek B., Kumar Varun S., 2015 "Mask-Less Electrochemical Additive Manufacturing: A Feasibility Study", ASME J. Manuf. Sci. Eng.

[8] Volgina V.M., Kabanovab T.B., Davydovb A.D., 2018 "Modeling of local maskless electrochemical deposition of metal microcolumns", Chemical Engineering Science (2018) 